http://jmscr.igmpublication.org/home/ ISSN (e)-2347-176x ISSN (p) 2455-0450 crossref DOI: https://dx.doi.org/10.18535/jmscr/v8i4.34

\title{
Outcomes of Lower Limb Ulcers in Diabetics vs Non Diabetics - Our Experience in a Tertiary Care Hospital
}

\author{
Authors \\ Pratha Anantha Ramani ${ }^{1}$, Chaitanya Babu Bogarapu ${ }^{2}$, Murali Manohar Deevi ${ }^{3}$, \\ Simhadri Uday Kiran ${ }^{4}$, Ginni Vijay Sainath Reddy ${ }^{5}$, Ginjupalli Saichand ${ }^{6}$, \\ Sivaram Shashank Yeeli ${ }^{7}$, Potireddy Yaswanth Reddy ${ }^{8}$ \\ ${ }^{1}$ Associate Professor, ${ }^{1}$ Assistant Professor, ${ }^{3,4,5,6,7,8}$ Post Graduate \\ Andhra Medical College, Visakhapatnam, India
}

\begin{abstract}
Background: Ulcers of lower extremities are debilitating to the patient directly and also have a great impact on the economy. The present study aims to examine the outcomes of lower limb ulcers, mainly in terms of amputations and mortality in the diabetic and non-diabetic population.

Research Design and Methods: A retrospective database containing 100 cases of lower limb ulcers from 2016 to 2017 were evaluated. History obtained and baseline clinical examination was done in all patients. Treatment options like debridement, fasciotomy, split-skin grafting, minor and major amputations were considered and outcomes studied.

Results: Majority of the patients were from rural areas with a mean age of 52 years. Males were the predominant study subjects. The ratio of diabetics to non-diabetics is 52:48. Debridement was done in 63\% of the cases, skin grafting in 13\%, fasciotomy in 6\%, minor amputations in $8 \%$ and major amputation in $10 \%$ of the study group. Above-knee amputation was done in 6 cases, of which only one was diabetic. 4 patients underwent below-knee amputation, of which 3 patients were diabetic. There were a total of 5 deaths, all of them being male, diabetic cases.

Conclusion: Diabetes has an increased risk of mortality and morbidity in patients with lower limb ulcers and also leads to significant impairment of quality of life. An aggressive multidisciplinary approach is required to manage foot problems in such patients and also to reduce the risk of death from other comorbid conditions to save both limb and life.
\end{abstract}

\section{Background}

"An ulcer is a discontinuity in a bodily membrane that impedes the organ of which the membrane is a part of from continuing its normal functions" $"(1)$. Ulcers of lower extremities are debilitating to the patient directly and also have a high impact on the economy since significant recourses are spent every year to treat, prevent, or decelerate the progression of the disease. There are many types of lower-limb ulceration of which venous, arterial, neurotrophic ulcers form the significant share. Ulcer types may differ in diabetics when compared to non-diabetics. Most of the diabetic patients have neuropathic ulcers, whereas nondiabetic patients are more likely to have venous, pressure or traumatic type ulcers ${ }^{(2)}$. Diabetes is a major risk factor for ulceration in the extremities, which posses significant mortality and morbidity. 
The present study aims to examine the outcomes of lower limb ulcers, mainly in terms of amputations and mortality in the diabetic and nondiabetic population.

\section{Research Design and Methods}

A retrospective database containing 100 cases of lower limb ulcers from 2016 to 2017, which were inpatients of King George Hospital, Visakhapatnam were evaluated. Baseline clinical examination was done to classify the ulcers. Some parameters such as age, gender, duration, average stay, diabetic status and its treatment status, seropositive status, cultures, organisms and outcomes were studied. Cultures were sent every $3^{\text {rd }}$ day. Treatment options like debridement, fasciotomy, split-skin grafting, minor and major amputations were considered.

A foot ulcer was defined as a full-thickness skin defect regardless of the duration. A standard neurological examination was done to test the sensation to light touch, pain, vibration and tendon reflexes at the ankle. Peripheral neuropathy was considered to be present if three of the four were absent. When both the dorsalis pedis and posterior tibial pulses were absent in the affected limb Peripheral vascular disease (PVD) was considered present. Based on this, ulcers were classified as neuropathic, ischemic, or neuro-ischemic.

\section{Results}

Among the 100 patients, 52\% are diabetic, and $48 \%$ are non-diabetic. Male and female patients are $72 \%$ and $28 \%$ respectively. There is one NACO positive male case, who is non-diabetic. Diabetic population presented from 18-65 years of age with an average of 46 years. Non-diabetic population presented from 24-90 years of age with an average of 57 years. The length of stay ranged from 3 days to 78 days in diabetics and 5 days to 90 days in non-diabetics.
Of the total patients in the study, $82 \%$ belong to the rural background, and $18 \%$ came from urban areas. 70 of 82 patients from rural areas and 4 patients from the urban population were illiterates. The size of the ulcer ranged from $3 \mathrm{~cm}$ to $40 \mathrm{~cm}$ in its greatest dimension, with an average being $12 \mathrm{~cm}$. The depth of the ulcer ranged from $0.5 \mathrm{~cm}$ to $3 \mathrm{~cm}$, with an average of $1 \mathrm{~cm}$.

Cultures were sent/available in 90 cases, of which E.coli was isolated in 27 patients, Klebsiella in 24 patients, Pseudomonas in 20 patients, Staphylococcus in 4 and Streptococcus in 1 patient. The culture was sterile in 14 patients and was not sent/ not available in 10 patients.

The neurological examination and examination for peripheral vascular disease revealed that sensations were absent in 12 patients and decreased in 9 patients majority of them being diabetic whereas peripheral pulsations were absent in 6 patients and decreased in 12 patients, being almost equal in diabetics and non-diabetics.

Of the 52 diabetic patients in the study, 17 patients are on insulin therapy, 32 patients are on oral hypoglycaemic agents, and 3 patients were not on any treatment for diabetes.

Anaemia $(\mathrm{Hb}<10 \%)$ was found in 38 diabetic patients, of which 23 are male, and 15 are female patients. It was found in 20 non-diabetic cases of which male and female are 15 and 5 respectively. Hypertension (B.P > 140/90mmhg) was another co-morbid condition found 32 diabetic patients, of whom 25 were male, and 7 were females. It was found to be present in 24 non-diabetics, of which 18 were male, and 6 were female patients. 


\section{JMSCR Vol||08||Issue ||04||Page 173-179||April}
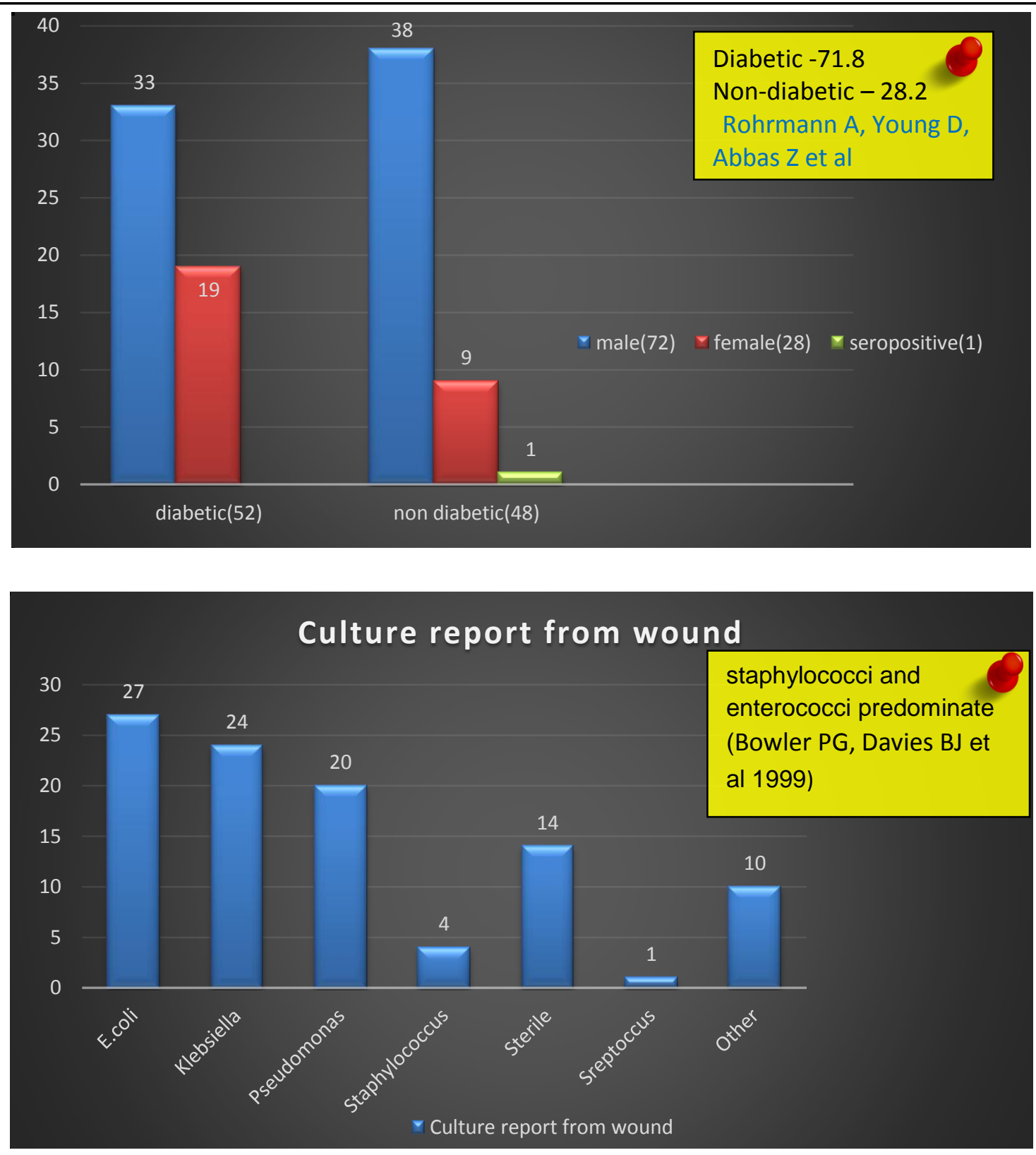

\section{Treatment in Diabetics}

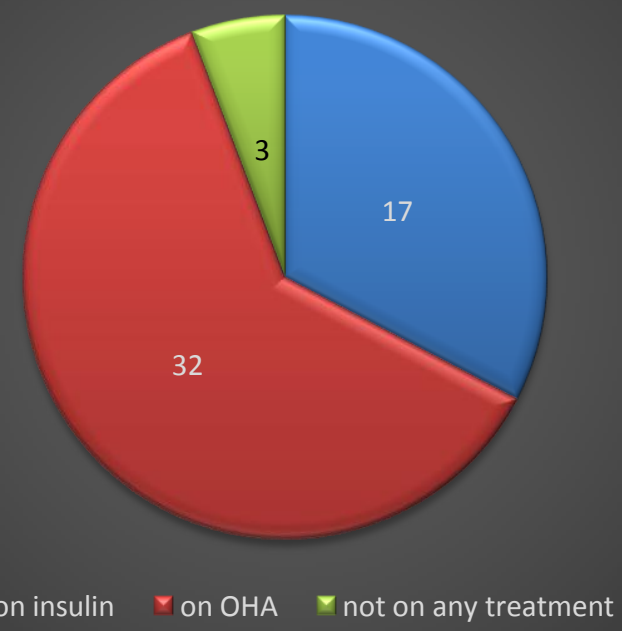




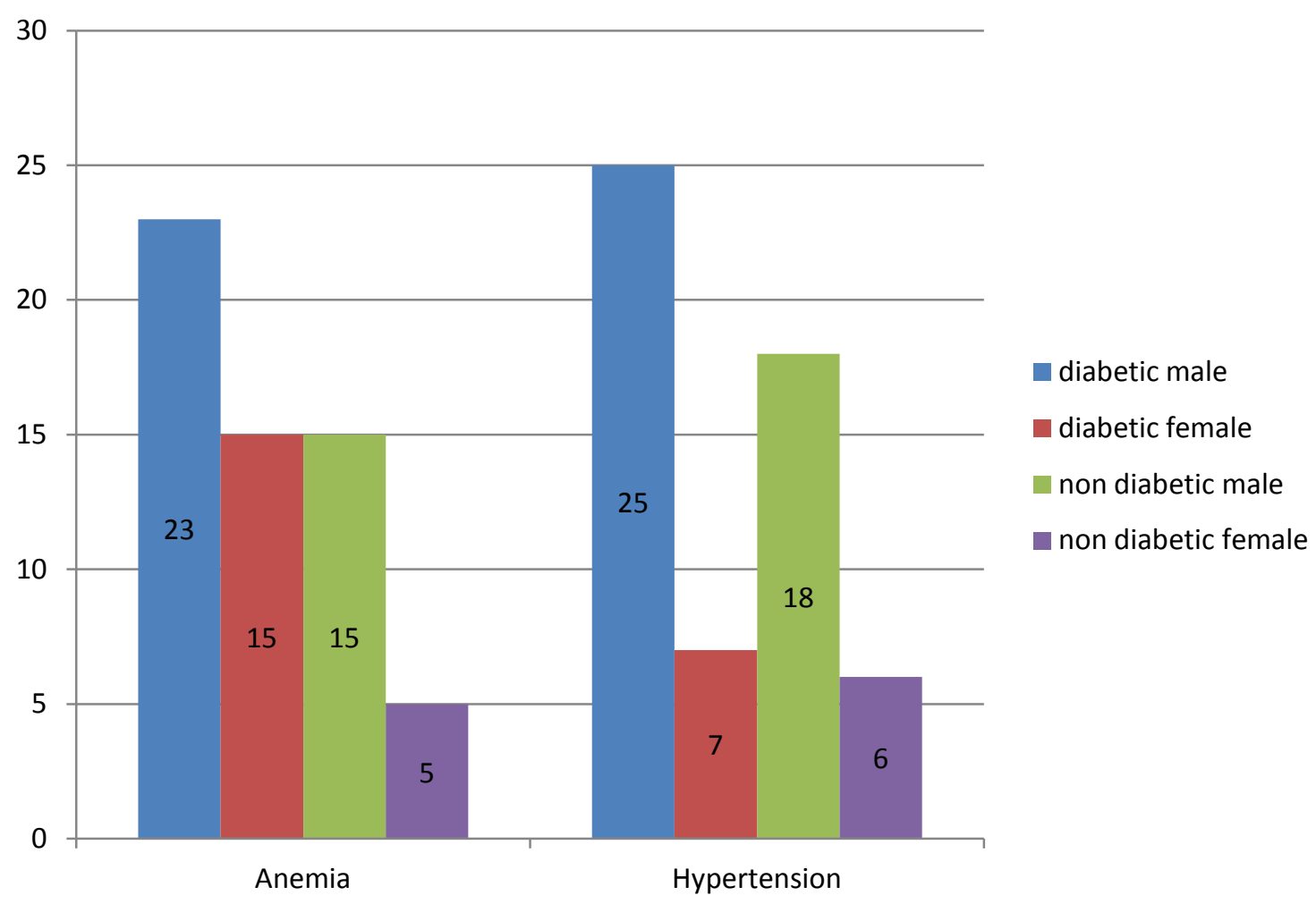

Regular debridement alone was satisfactory in $63 \%$ of the study group whereas debridement along with skin grafting was required in $13 \%$ and fasciotomy in $6 \%$ of the patients. Minor amputations were carried out in $8 \%$ and major amputation in $10 \%$ of the study group. Above- knee amputation was required in 6 cases, of which only one patient was diabetic. 4 patients underwent below-knee amputation, of which 3 patients were diabetic which includes one death. Readmission was required in $21 \%$ of the study population.

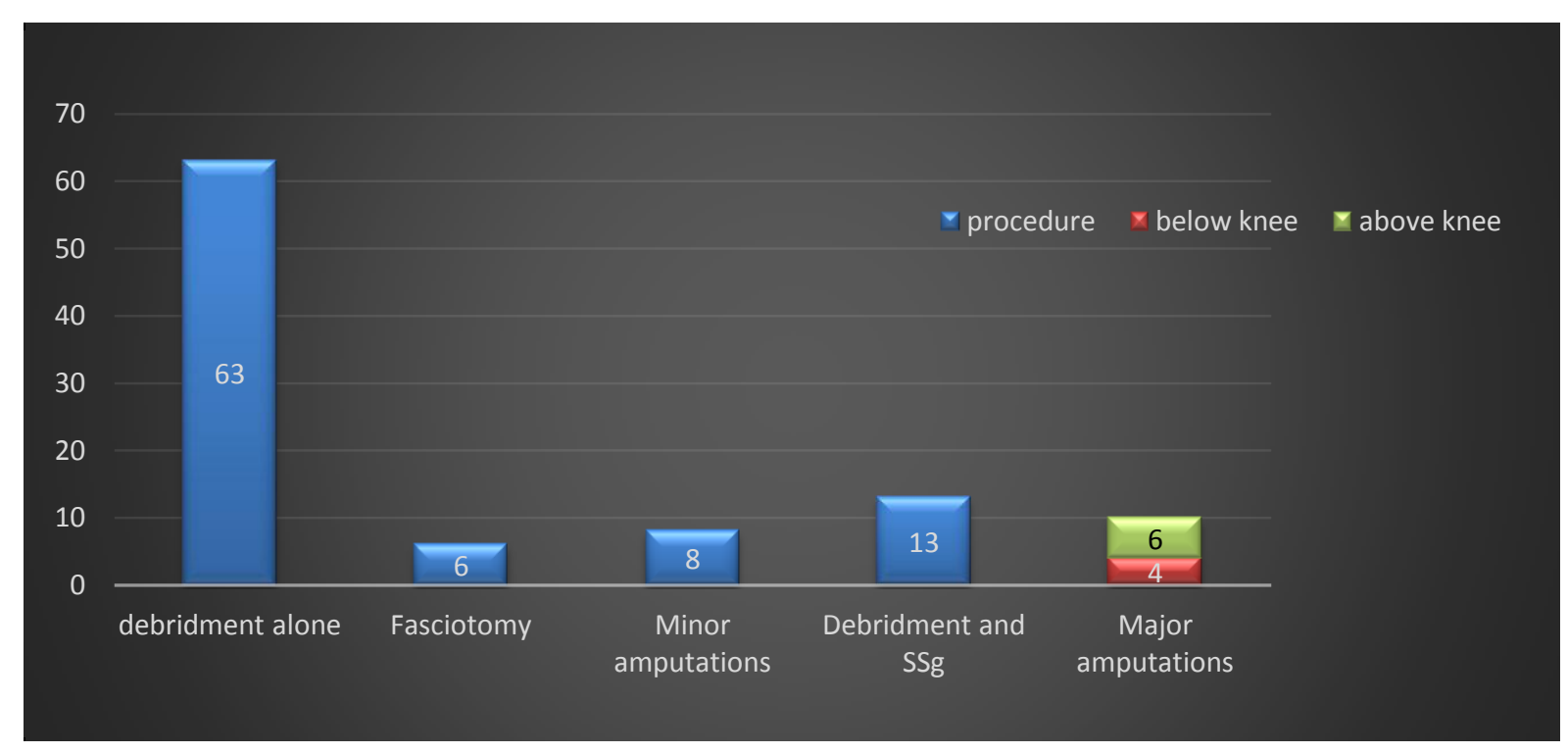




\section{JMSCR Vol||08||Issue ||04||Page 173-179||April}

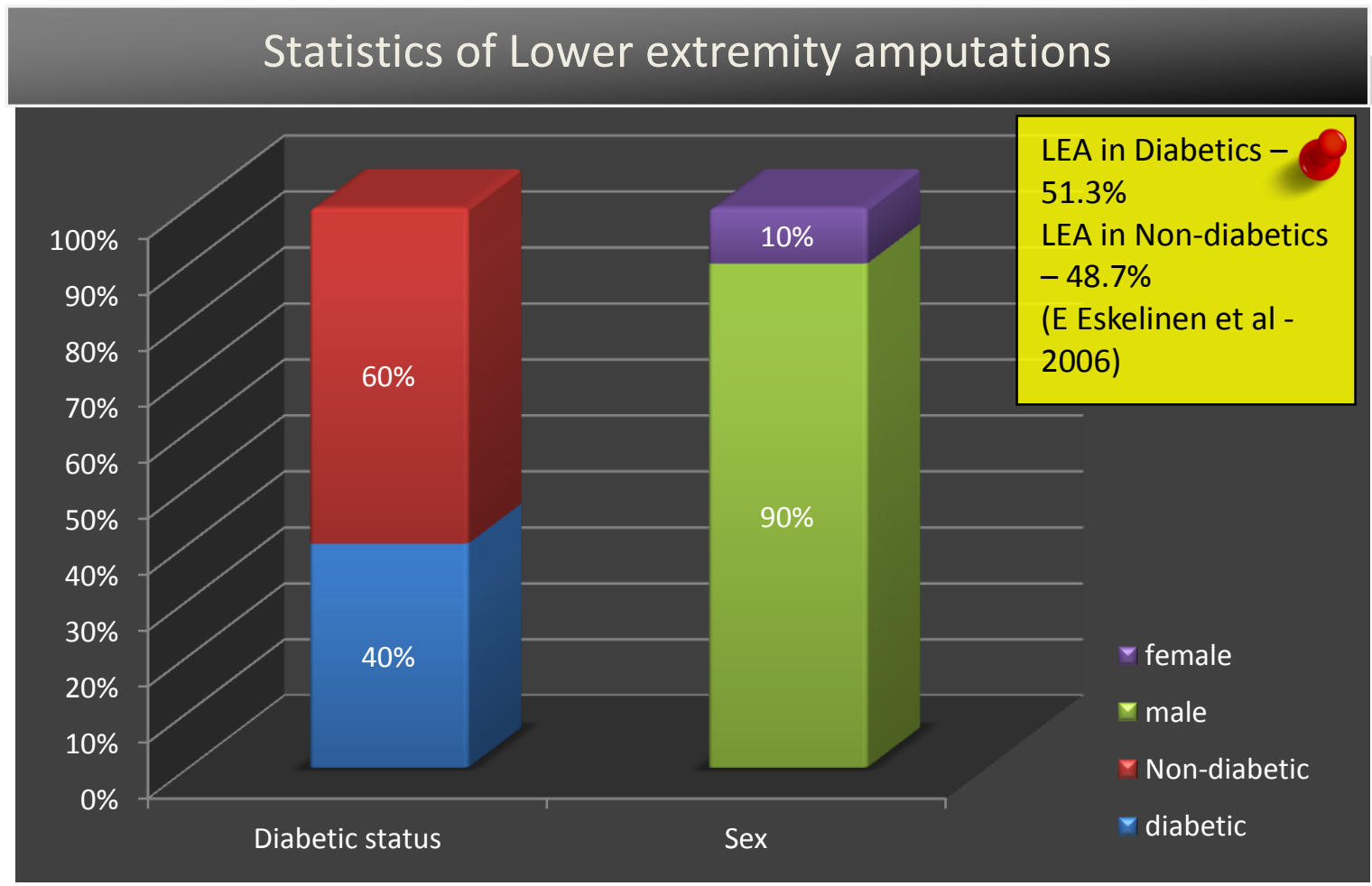

There were a total of 5 deaths recorded, all of them being diabetic and belonging to the male sex. The mean age was around 60 years. The duration of ulcers in these patients ranged from 2 days to 45 days and the length of stay in hospital was less than 5 days.

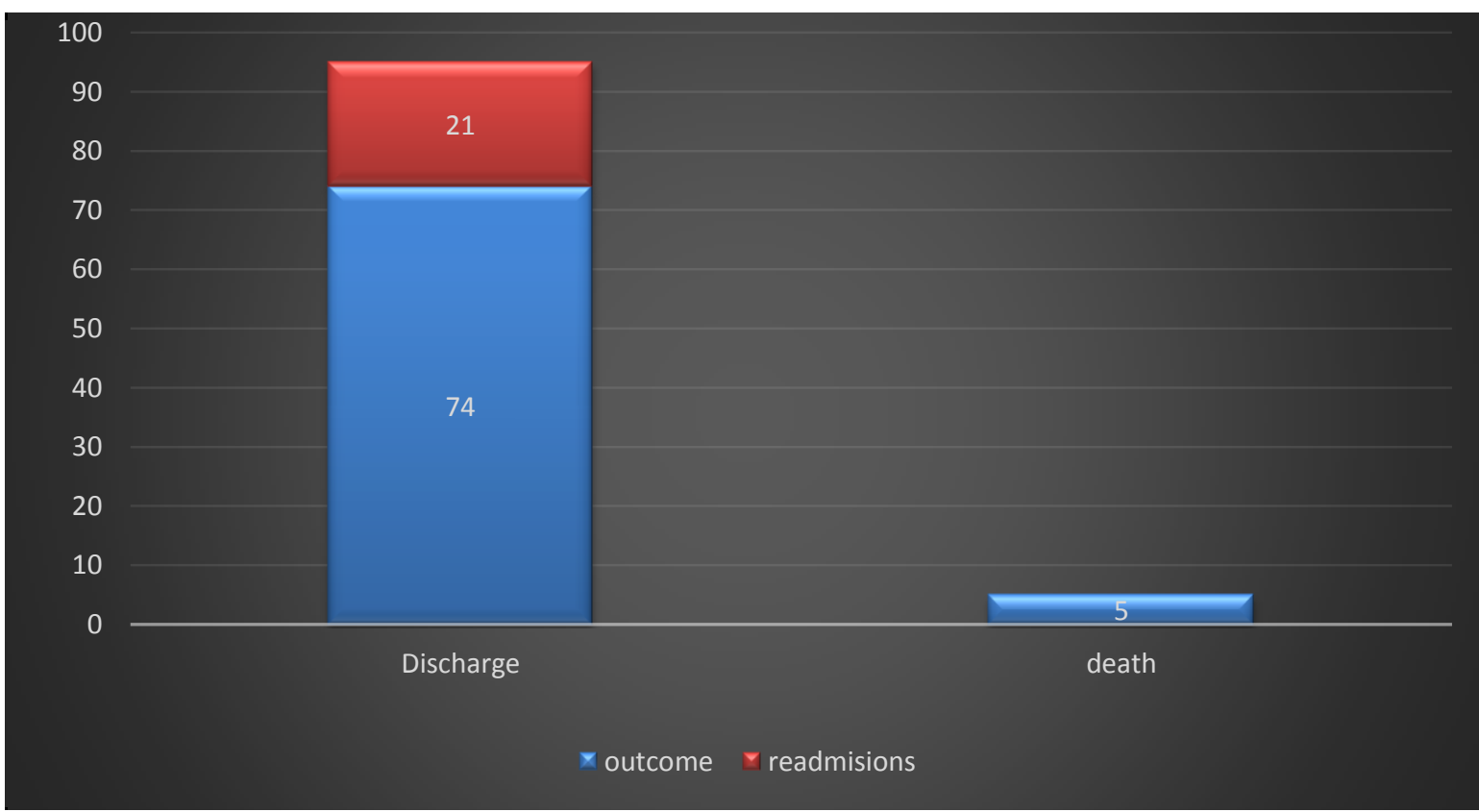




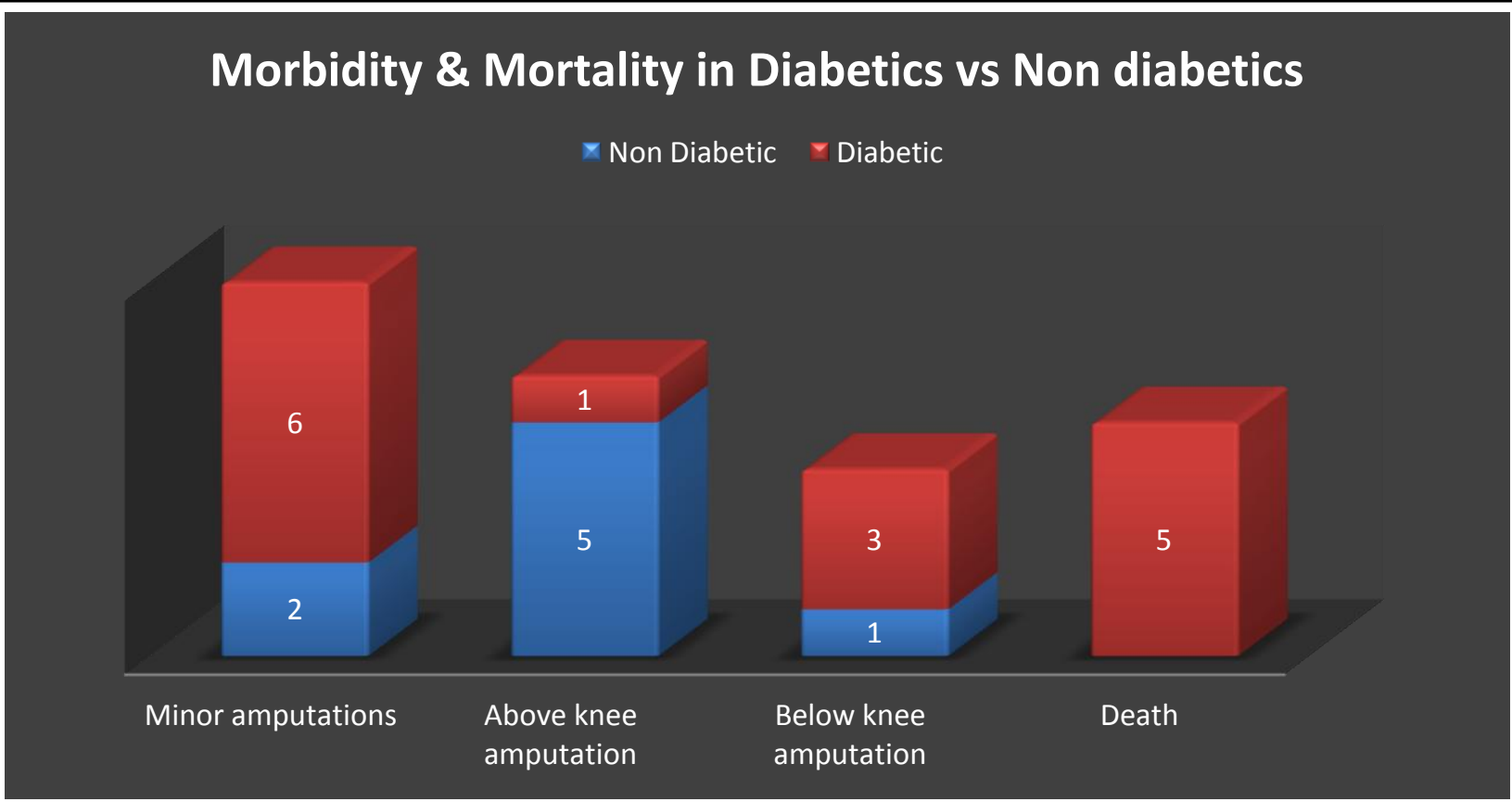

\section{Discussion}

In our study, most of the patients were from rural areas with an age range from 18 to 90 years and a mean age of 52 years. Males were the predominant study subjects. The ratio of patients with and without diabetes is almost equal (52:48) in our study which differs from many national and international studies where diabetics predominate - [Rohrmann A, Young D, Abbas Z et al. $(71.8: 28.2)]^{(2)}$. On average, diabetic patients were younger than non- diabetics (average age $46 \mathrm{y}$ in diabetics, $57 \mathrm{y}$ in non-diabetics). $82 \%$ of the cases belonging to the rural population and an overall $74 \%$ illiteracy signify the importance of health education that needs to be addressed. Neuropathic ulcers were predominant in diabetics, whereas other types of ulcers were seen more with nondiabetics population, which correlates with the literature (Spentzouris and Labropoulos 2009) ${ }^{(3)}$. The culture and sensitivity burden in our study was carried mainly by E.coli, Klebsiella and Pseudomonas which varies with other studies where staphylococci and enterococci predominate (Bowler PG, Davies BJ et al. 1999) $^{(5)}$. Majority of the diabetic population has other co-morbid conditions such as anaemia and hypertension, the percentage being more in females $(\sim 70 \%)$. Poor diabetic control was found in 20 of 52 cases with diabetes. The incidence of Lower extremity amputations was less in diabetics $(40 \%)^{(8)}$. Though the number of major amputations was slightly more in non-diabetics in our study, a significant proportion of minor amputations being carried out in diabetics and the total death toll $(5 \%)$ belonging only to diabetic population signifies the higher risk of mortality and morbidity associated with diabetes in lower limb ulcers. The mortality appears to be independent of factors increasing the ulcer risk, i.e., neuropathy and PVD (N. K. Chammas, R. L. R. Hill, M. E. Edmonds - J 2016) (4).

\section{Conclusion}

Diabetes has an increased risk of mortality and morbidity in patients with lower limb ulcers and also leads to significant impairment of quality of life. Poor control of diabetes found in nearly half of the diabetic patients emphasizes the need for specialized diabetic units. An aggressive multidisciplinary approach is required to manage foot problems in such patients and also to recognize and reduce the risk of death from other co-morbid conditions to save both limb and life.

Emphasis should be made on educating people about diabetes and its complications, foot care and wound care to reduce the burden of lower limb ulcers and to improve the quality of life. 


\section{References}

1. Wikipedia https://en.wikipedia.org/wiki/Ulcer

2. Rohrmann A, Young D, Abbas Z et al. (2013) Profile of diabetic and non-diabetic patients treated for wounds or ulcers. Diabetic Foot Canada 1(2): 42-7

3. Georgios Spentzouris and Nicos Labropoulos et al.- The Evaluation of Lower-Extremity Ulcers

4. N. K. Chammas, R. L. R. Hill, M. E. Edmonds - J - Increased Mortality in Diabetic Foot Ulcer Patients: The Significance of Ulcer Type. Diabetes Res. 2016; 2016: 2879809. Published online 2016 Apr 24. DOI: $10.1155 / 2016 / 2879809$ PMCID: PMC4860228.

5. Bowler PG, Davies_BJ. - The microbiology of infected and non-infected leg ulcers. PMID: 10487444

6. Tesfamichael G. Mariam, Abebaw Alemayehu, Eleni Tesfaye, Worku Mequannt, Kiber Temesgen, Fisseha Yetwale, Miteku Andualem Limenih - Prevalence of Diabetic Foot Ulcer and Associated Factors among Adult Diabetic Patients Who Attend the Diabetic Follow-Up Clinic at the University of Gondar Referral Hospital, North West Ethiopia, 2016: Institutional-Based CrossSectional Study- J Diabetes Res. 2017; 2017: 2879249. Published online 2017 Jul 16. DOI: $10.1155 / 2017 / 2879249$.

\section{PMCID: PMC5534295}

7. Probal K. Moulik, Robert Mtonga and Geoffrey V. Gill - Amputation and Mortality in New-Onset Diabetic Foot Ulcers Stratified by Etiology. Diabetes Care 2003 Feb; 26 (2): 491-494. https://doi.org/10.2337/diacare.26.2.491

8. Eskelinen, E., Eskelinen, A., Albäck, A., \& Lepäntalo, M. (2006). Major Amputation Incidence Decreases Both in Non-Diabetic and in Diabetic Patients in Helsinki.
Scandinavian Journal of Surgery, 95(3), 185 189. doi:10.1177/145749690609500311

9. Fosse S, Hartemann-Heurtier A, et al. Incidence and characteristics of lower-limb amputations in people with diabetes. Diabetic Medicine. 2009; 26: 391-396.

10. Renzi R, Unwin N, Jubelirer R, et al. An international comparison of lower extremity amputation rates. Ann Vasc Surg. 2006;20 (3):346-350.

11. Maria Narres, Tatjana Kvitkina, Heiner Claessen, Sigrid Droste, Björn Schuster, Stephan Morbach, Gerhard Rümenapf, Kristien Van Acker, Andrea Icks - Incidence of lower extremity amputations in the diabetic compared with the non-diabetic population: A systematic review. PLoSOne.2017; 12(8):e0182081.

DOI: 10.1371/journal.pone.0182081.PMCID: PMC5573217

12. Jakob C. Thorud, DPM, MS, FACFAS, and Joslin L. Seidel, BS - A closer look at mortality after lower extremity amputation.

13. Rizk MN, Ameen AI. Comorbidities associated with Egyptian diabetic foot disease subtypes. Egypt J Intern Med 2013;25:154-8

14. Ingrid Kruse, DPM and Steven Edelman, MD - Evaluation and Treatment of Diabetic Foot Ulcers Clinical Diabetes 2006 Apr; 24 (2): 9193. https://doi.org/10.2337/diaclin.24.2.91. 